\title{
Pneumomediastinum, pneumopericardium pneumothorax and subcutaneous emphysema in Iranian COVID-19 patients
}

\author{
Manouchehr Aghajanzadeh ${ }^{1}$, Mohammad Haghighi2,,*, Siamak Rimaz², Ali Alavi Fomani3, Azita
}

Tangestaninejad3, Mohammad Taghi Ashoobi3 3 , Yousha Pourahmadi3 ${ }^{3}$, Mohammad Mojtaba Rohani4

'Department of Thoracic Surgery, Guilan University of Medical Sciences, Rasht, Iran

${ }^{2}$ Anesthesiology Research Center, Department of Anesthesiology, Alzahra Hospital, Guilan University of Medical Sciences, Rasht, Iran

3Inflammatory Lung Diseases Research Center, Department of Internal Medicine, Razi Hospital, School of Medicine, Guilan University of Medical Sciences, Rasht, Iran

4Department of Radiology, Razi Hospital, Guilan University of Medical Science, Rasht, Iran

\begin{abstract}
Recently, spontaneous pneumomediastinum (PM), pneumopericardium (PP), pneumothorax (PT), and subcutaneous emphysema (SE) were reported as infrequent complications in coronavirus disease 2019 (COVID-19) patients in intensive care (ICU). Here we report these complications in nine Iranian patients of COVID-19. Nine patients with reported PM, PP, PT, and SE in COVID-19 who were hospitalized in Arya hospital, Rasht, Iran, for three months, were followed to record demographic data and clinical characteristics of these patients. In nine PM-developed patients, six cases represented PT, one patient with PP, and four patients with PT and SE. Four patients expired and only five patients survived. PM, PP, PT, and SE are uncommon complications in COVID-19 patients and were reported frequently in male patients. Early diagnosis and treatment could save the patients since these complications are related to poor prognosis and prolonged hospitalization. Patients with mild COVID19 and mild pulmonary damage have a favorable outcome.
\end{abstract}

Keywords: COVID-19, Subcutaneous emphysema, Spontaneous pneumomediastinum, Pneumopericardium, Pneumothorax

\section{Introduction}

Severe acute respiratory syndrome coronavirus-2 (SARS-CoV-2) infection has developed as the greatest pandemic in this era with high morbidity and mortality and multiple complications. There are lots of review studies about coronavirus disease 2019 (COVID-19) and its complications [1-4]. Irregular reported numbers of spontaneous pneumomediastinum (PM), pneumopericardium (PP), pneumothorax (PT), and subcutaneous emphysema (SE) cases in patients with COVID -19, and also in the patients without mechanical ventilation, which results in barotrauma [5]. The pathophysiologic mechanism of PM is an increased gradient pressure between the alveoli and parenchyma of lung that leads to severe alveolar injury and the air dissects the surrounding bronchovascular sheaths, and this air enters into the mediastinum and produces PM and disturbance to cervical SE and pleural space PT $[3,5,6]$. Alveolar wall is more prone to rupture due to inflammation in COVID-19 patients and alveolar wall is exacerbated by severe cough or any problems which increase the intra-alveolar pressure [5]. Invasive positive pressure ventilation can cause

\section{*Corresponding author:}

Mohammad Haghighi, MD

Department of Anesthesiology, Alzahra Hospital, Guilan University of Medical Sciences, Rasht, Iran

Tel/Fax: +98 9113315256

Email: manesthesist@gmail.com

http://orcid.org/o000-0003-0331-1683

Received: September, 15, 2021

(C) The Author(s) 2021

Accepted: December, 14, 2021 
PT and/or PM spontaneously. Barotrauma is the cause of PM, PT, PP, and SE [3]. In this care report, we focus only on hospitalized COVID-19 patients who developed these complications. Some of patients have primarily mild to moderate disease condition, but deteriorated progressively, and also represented the associated factors to prognosis these complications.

\section{Case presentation}

In this case report, we evaluated case series and described the record of nine patients in Arya hospital, Rasht, Iran during three months in COVID-19 fourth outbreak from April until July 2020. Patients demographic data, medical histories, clinical and laboratory finding, chest computer tomography (CT) results, and patient's outcome (death or discharged), were recorded. All subjects gave their informed consent to participate in the study.

According to our evaluation, seven out of nine patients with COVID-19 were male and two of them were female. The age range was about 32 to 68 years by mean of $48.27 \pm 10$. Diagnosis of COVID-19 was confirmed by polymerase chain reaction (PCR). Among nine patients, three of them reported the history of hypertension, four of them had type 2 diabetes mellitus, two patients had bronchial asthma, and also two of them had obesity. After two to six days of hospitalization, all of the nine patients represented one or more following complications: PM, PP, PT, and SE. Three patients with low oxygen saturation (between $50 \%$ and $86 \%$ ), improved up to $90-93 \%$ on BiPAP mask at $12 \mathrm{~L} / \mathrm{min}$. Chest CT was done to evaluate the severity of lung damaged due to SARS$\mathrm{CoV}-2$ at the early stage (Supplementary Figures $1-8$ ). The most common radiologic findings were groundglass pattern, integrated opacity, and thickening of septum. Four patients suffering minor pulmonary damage (10-35\% of lung), three of them had moderate injury (50\% of lung) and four cases had severe forms of injury (over $70 \%$ of lung). None of patients represented PM, PP, PT, or SE complications, while these complications happened after 1 to 8 days of hospitalization.

Laboratory findings have revealed following data: the count of neutrophil increased but lymphocyte decreased, $\mathrm{C}$ reactive protein (CRP) was higher than $100 \mathrm{mg} / \mathrm{L}$, also the levels of ferritin and lactate were increased. Arterial blood gases evaluation was as follows: the mean of initially $\mathrm{PO}_{2}$ was $78 \mathrm{mmHg}$ (59-
83), but later decreased to $54 \mathrm{mmHg}(48-85)$. The mean of initially $\mathrm{PCO}_{2}$ was $60 \mathrm{mmHg}(48-68)$, and the mean of and $\mathrm{O}_{2}$ saturation was $80.2 \%(60-98 \%)$. All patients were received one ceftriaxone course of treatment in combination with remdesivir, dexamethasone, and levofloxacin. Cardiothoracic surgeon consulted all cases. Throughout hospitalization, eight patients needed oxygen therapy, six patients were transferred to the intensive care unit (ICU), and underwent invasive ventilation. Six patients needed chest tube, and six patients with PT needed subclavicular incision for evacuation of SE, while the rest of them were managed conventionally. Four patients died after 8-20 days, with the median of 6-12 days of hospitalization. The remaining five patients who had moderate lung damage at admission time, did not need surgical drainage and were discharged in good clinical condition after hospitalization (8-12 days). Clinical characteristics of all patients were explained in detail in Table 1.

\section{Discussion}

PM is presented in two types, benign or malignant, which in some patient it can be fatal [7]. Benign type of PM can turn to malignant forms, in the case of rapidly rise pressure in the mediastinum, which causes all spaces to closed with no way for air escape. Malignant PM could consequence mechanical obstruction and presents as pericardial tampon, especially in the present of cough. This event can interfere with the cardiovascular function and causes reduction in circulation volume and tension PT form $[5,8,9]$, which none of the nine reported patients represented this complication. In some patients with PM, air can escape into route to the neck, retroperitoneum or to the right and left pleural space, and causes bilateral PT, which can turn into tension PT form [10]. A retrospective study has reported that 13 patients with COVID-19 have developed SPM with or without PT and SE [5, 8, 9]. In this case report, no patient had isolated PT and some of these patients needed invasive positive pressure ventilation. Furthermore, none of reported patients have represent with retroperitoneal emphysema. Moreover, this case report has showed high level serum of $\mathrm{LDH}$, which might be due to cellular damage. Also, severe diffuse alveolar damage causes of the alveolar damage, and air escape along the bronchoalveolar sheath and mediastinum space [8]. 


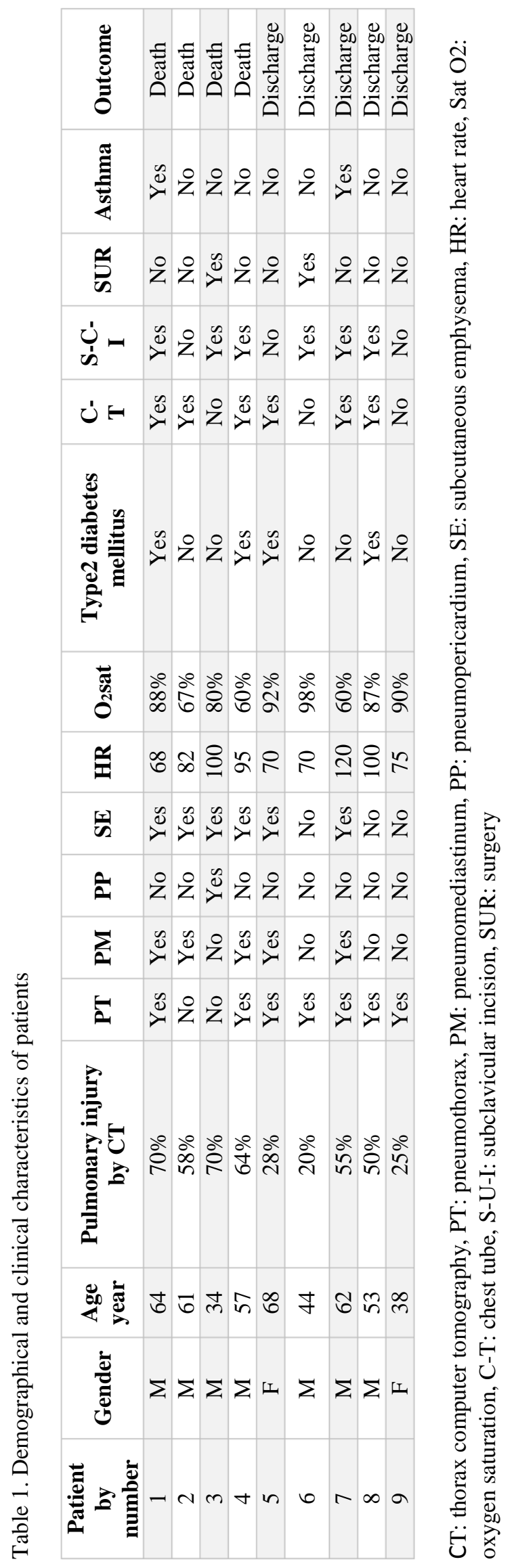


A similar study, reported COIVD-19 patients that represented pneumothorax (PTX), PM, and SE spontaneously or after mechanical ventilation [11]. We observed the occurrence of these complications in nine patients. In these reported patients, four of them represented spontaneously complications without invasive mechanical ventilation, while other represented complications in patients with invasive mechanical ventilation. As reported in other studies with the majority of male gender, seven of patients were male and two of them were female. The pathophysiological mechanism of complications was air leakage through damage of alveolar walls by inflammation during of cytokine storm [5, 12-14]. Despite to some studies with low mortality rate, in this case report, patient's mortality rate due to PM, PP, PT, and SE was four out of nine patients.

PM, PP, PT, and SE are rare complications in severe COVID-19 pneumonia.These complications are contributed to poor prognosis and prolonged hospitalization. Patients with mild COVID-19 and mild pulmonary damage have a favorable outcome.

\section{Supplementary files}

Supplementary file 1 .

\section{Acknowledgments}

We would like to thank all individuals who volunteered to participate in this study. Furthermore, thank you to hospital staff and specialists for assistance with conforming and recording cases.

\section{Authors' contribution}

Concept and Study design: MA, MH, SR; Methods and experimental work: AA, AT, MTA; Results analysis and conclusions: MA, MH, YP; Manuscript preparation: MMR, AA, AT, MTA. All authors read and approved the final version.

\section{Conflict of interests}

No potential conflict of interest was reported by the authors.

\section{Ethical declarations}

All subjects gave their informed consent to participate in the study. Also, all methods were performed in accordance with the relevant guidelines and regulations.

\section{Consent for publication}

Written informed consent was obtained from the patients for publication of this case report. A copy of the written consent is available for review by the Editor-in-Chief of this journal.

\section{Availability of data and material}

Corresponding author (Mohammad Haghighi) is available in the case in requiring, and all data and tables, used in the manuscript are prepared originally by authors

\section{Financial Support}

Self-funded.

\section{References}

1. Hamad AM, Elmahrouk AF, Abdulatty OA. Alveolar air leakage in COVID-19 patients: Pneumomediastinum and/or pneumopericardium. Heart Lung. 2020; 49(6):881-2.

2. Murillo Brito D, Villalva C, Simón A, Guisado-Clavero M. COVID-19 pneumonia associated with spontaneous pneumomediastinum and pneumopericardium. CTSNET. 2020:12485831.

3. Martinelli AW, Ingle T, Newman J, Nadeem I, Jackson K, Lane ND, et al. COVID-19 and pneumothorax: a multicentre retrospective case series. Eur Respir J. 2020; 56(5):2002697. 4. Elhakim TS, Abdul HS, Pelaez Romero C, Rodriguez-Fuentes Y. Spontaneous pneumomediastinum, pneumothorax and subcutaneous emphysema in COVID-19 pneumonia: a rare case and literature review. BMJ Case Rep. 2020; 13(12):e239489.

5. Juárez-Lloclla JP, León-Jiménez F, Urquiaga-Calderón J, Temoche-Nizama H, Bryce-Alberti M, Portmann-Baracco A, et al. Spontaneous Pneumopericardium and Pneumomediastinum in Twelve COVID-19 Patients. Arch Bronconeumol. 2021; 57:86-8. 6. Aghajanzadeh M, Dehnadi A, Ebrahimi H, Fallah Karkan M, Khajeh Jahromi S, Amir Maafi A, et al. Classification and Management of Subcutaneous Emphysema: a 10-Year Experience. Indian J Surg. 2015; 77(Suppl 2):673-7.

7. Macklin MT, Macklin C. Malignant interstitial emphysema of the lungs and mediastinum as an important occult complication in many respiratory diseases and other conditions. 1944:281-358. 8. Jougon JB, Ballester M, Delcambre F, Mac Bride T, Dromer CE, Velly JF. Assessment of spontaneous pneumomediastinum: experience with 12 patients. Ann Thorac Surg. 2003; 75(6):1711-4. 9. Sun R, Liu H. Mediastinal Emphysema, Giant Bulla, and Pneumothorax Developed during the Course of COVID-19 Pneumonia. Korean J Radiol. 2020; 21(5):541-4.

10. Chu CM, Leung YY, Hui JY, Hung IF, Chan VL, Leung WS, et al. Spontaneous pneumomediastinum in patients with severe acute respiratory syndrome. Eur Respir J. 2004; 23(6):802-4.

11. Wadhawa R, Thakkar A, Chhanwal HS, Bhalotra A, Rana Y, Wadhawa V. Spontaneous pneumomediastinum and subcutaneous emphysema in patients with COVID-19. Saudi $J$ Anaesth. 2021; 15(2):93-6. 


\section{Aghajanzadeh et al.}

12. Mason RJ, Slutsky A, Murray JF, Nadel JA, Gotway MB. Murray \& Nadel's Textbook of Respiratory Medicine E-Book: Elsevier Health Sciences; 2015.

13. Vancheri SG, Savietto G, Ballati F, Maggi A, Canino C, Bortolotto C, et al. Radiographic findings in 240 patients with COVID-19 pneumonia: time-dependence after the onset of symptoms. Eur Radiol. 2020; 30(11):6161-9.
14. Kolani S, Houari N, Haloua M, Alaoui Lamrani Y, Boubbou M, Serraj M, et al. Spontaneous pneumomediastinum occurring in the SARS-COV-2 infection. IDCases. 2020; 21:e00806. 